\title{
EXTENDED A CONSTANT PART OF REDHEFFER'S TYPE INEQUALITIES
}

\author{
YUSUKE NISHIZAWA
}

Abstract. J-L. Li and Y-L. Li [4] gave the following Redheffer's type inequality;

$$
\frac{1-\left(\frac{x}{\pi}\right)^{2}}{\sqrt{1+3\left(\frac{x}{\pi}\right)^{4}}}>\frac{\sin x}{x}
$$

holds for $0<x<\pi$, where the constant 3 is the best possible. In this paper, we establish two inequalities extended the constant part of the above inequality.

\section{Introduction}

Redheffer et al. [7], [8] established the following inequality; the inequality

$$
\frac{\sin x}{x} \geq \frac{\pi^{2}-x^{2}}{\pi^{2}+x^{2}}
$$

holds for $0<x \leq \pi$. After that, mathematicians [1]-[4], [6]-[11] studied the Redheffer's type inequalities. Chen et al. [2] gave the Redheffer's type inequalities for $\cos x$ and $\frac{\sinh x}{x}$, Baricz et al. [1], [6], [11] established the Redheffer's type inequalities extended for Bessel functions and Zhu et al. [9] [10] showed the Redheffer's type inequalities for circular and hyperbolic functions. Especially, Li et al. [4] showed the following simple Redheffer's type inequality; the inequality

$$
\frac{1-\left(\frac{x}{\pi}\right)^{2}}{\sqrt{1+3\left(\frac{x}{\pi}\right)^{4}}}>\frac{\sin x}{x}
$$

holds for $0<x<\pi$, where the constant 3 is the best possible. In this papar, we consider the above inequality (1.2) and our main results are followings.

Received June 19, 2017, accepted August 16, 2017.

2010 Mathematics Subject Classification. Primary: 26D05, 26 D07.

Key words and phrases. Redheffer's inequalities, monotonically increasing functions, monotonically decreasing functions, trigonometric functions. 
Theorem 1.1. For $r>3$ and $0<x<\pi-\pi \sqrt{\frac{r-3}{r}}$, we have

$$
\frac{1-\left(\frac{x}{\pi}\right)^{2}}{\sqrt{1+r\left(\frac{x}{\pi}\right)^{4}}}>\frac{\sin x}{x} \text {. }
$$

The above inequality (1.3) is extended the constant part of the inequality (1.2).

Theorem 1.2. For $r>3$ and $\pi \sqrt{\frac{2}{r-1}}<x<\pi$, we have

$$
\frac{\sin x}{x}>\frac{1-\left(\frac{x}{\pi}\right)^{2}}{\sqrt{1+r\left(\frac{x}{\pi}\right)^{4}}} .
$$

The above inequality (1.4) is extended the constant part and the reversed type of the inequality (1.2).

Remark 1.3. It seem likely to that the inequality (1.3) holds for $r>3$ and $0<x<\pi-\pi \sqrt{\frac{r-3}{r+5}}$, which is a stronger than the condition of Theorem 1.1.

\section{Proof of main theorems}

Proof of Theorem 1.1. From $0<x<\pi-\pi \sqrt{\frac{r-3}{r}}$, we have

$$
3<r<\frac{3 \pi^{2}}{(2 \pi-x) x}
$$

and

$$
\begin{aligned}
\frac{1-\left(\frac{x}{\pi}\right)^{2}}{\sqrt{1+r\left(\frac{x}{\pi}\right)^{4}}}-\frac{\sin x}{x} & >\frac{1-\left(\frac{x}{\pi}\right)^{2}}{\sqrt{1+\left(\frac{3 \pi^{2}}{(2 \pi-x) x}\right)\left(\frac{x}{\pi}\right)^{4}}}-\frac{\sin x}{x} \\
& =\sqrt{\frac{(\pi-x)^{2}(x+\pi)^{2}(2 \pi-x)}{\pi^{2}\left(3 x^{3}-\pi^{2} x+2 \pi^{3}\right)}}-\frac{\sin x}{x} .
\end{aligned}
$$

Hence, it suffices to show that

$$
F(x)=\frac{(\pi-x)^{2}(x+\pi)^{2}(2 \pi-x)}{\pi^{2}\left(3 x^{3}-\pi^{2} x+2 \pi^{3}\right)}-\frac{\sin ^{2} x}{x^{2}}>0 .
$$

First, we consider the case of $0<x \leq \frac{3 \pi}{4}$. In this case, the following inequality is the important role of the proof. Li [5] showed that

$$
\frac{x}{\sin x}=1+\sum_{n \in \mathbb{Z} \backslash 0} \frac{(-1)^{n+1}\left(\frac{x}{\pi}\right)^{2}}{n^{2}+n\left(\frac{x}{\pi}\right)}=1+2\left(\frac{x}{\pi}\right)^{2} \sum_{n=1}^{\infty} \frac{(-1)^{n+1}}{n^{2}-\left(\frac{x}{\pi}\right)^{2}}
$$


for $0<x<\pi$. It follows from the above inequality (2.1) that

$$
\frac{x}{\sin x} \geq 1+2\left(\frac{x}{\pi}\right)^{2}\left\{\frac{1}{1-\left(\frac{x}{\pi}\right)^{2}}-\frac{1}{4-\left(\frac{x}{\pi}\right)^{2}}\right\}
$$

for $0<x<\pi$. Thus, we can get

$$
\frac{\sin x}{x} \leq \frac{(\pi-x)(2 \pi-x)(x+\pi)(x+2 \pi)}{x^{4}+\pi^{2} x^{2}+4 \pi^{4}}
$$

for $0<x<\pi$ and we have

$$
\begin{aligned}
F(x) & \geq \frac{(\pi-x)^{2}(x+\pi)^{2}(2 \pi-x)}{\pi^{2}\left(3 x^{3}-\pi^{2} x+2 \pi^{3}\right)}-\left\{\frac{(\pi-x)(2 \pi-x)(x+\pi)(x+2 \pi)}{x^{4}+\pi^{2} x^{2}+4 \pi^{4}}\right\}^{2} \\
& =\frac{(\pi-x)^{3}(2 \pi-x) x^{2}(x+\pi) F_{1}(x)}{\pi^{2}\left(3 x^{2}-3 \pi x+2 \pi^{2}\right)\left(x^{4}+\pi^{2} x^{2}+4 \pi^{4}\right)^{2}},
\end{aligned}
$$

where $F_{1}(x)=-x^{5}-\pi x^{4}-6 \pi^{2} x^{3}-12 \pi^{3} x^{2}-8 \pi^{4} x+16 \pi^{5}$. Since $F_{1}(x)$ is strictly decreasing for $0<x<\frac{3 \pi}{4}$, we have

$$
F_{1}(x)>F_{1}\left(\frac{3 \pi}{4}\right)=\frac{169 \pi^{5}}{1024}>0
$$

and

$$
3 x^{2}-3 \pi x+2 \pi^{2} \geq 3\left(\frac{\pi}{2}\right)^{2}-3 \pi\left(\frac{\pi}{2}\right)+2 \pi^{2}=\frac{5 \pi^{2}}{4}>0 .
$$

Thus, we obtain $F(x)>0$ for $0<x \leq \frac{3 \pi}{4}$. Next, we consider the case of $\frac{3 \pi}{4}<x<\pi$. By Taylor series, we have

$$
\cos 2 x>1-2(x-\pi)^{2}+\frac{2}{3}(x-\pi)^{4}-\frac{4}{45}(x-\pi)^{6}
$$

for $\frac{3 \pi}{4}<x<\pi$, so we can get

$$
\begin{aligned}
F(x) & =\frac{-2 x^{6}+6 \pi x^{5}-2 \pi^{2} x^{4}-6 \pi^{3} x^{3}+4 \pi^{4} x^{2}-3 \pi^{2} x^{2}+3 \pi^{3} x-2 \pi^{4}}{2 \pi^{2} x^{2}\left(3 x^{2}-3 \pi x+2 \pi^{2}\right)}+\frac{\cos 2 x}{2 x^{2}} \\
& >\frac{(\pi-x)^{4} F_{2}(x)}{45 \pi^{2} x^{2}\left(3 x^{2}-3 \pi x+2 \pi^{2}\right)},
\end{aligned}
$$

where

$$
\begin{aligned}
F_{2}(x)= & -6 \pi^{2} x^{4}+18 \pi^{3} x^{3}-22 \pi^{4} x^{2}+45 \pi^{2} x^{2}-45 x^{2} \\
& +14 \pi^{5} x-45 \pi^{3} x-45 \pi x-4 \pi^{6}+30 \pi^{4}-90 \pi^{2} .
\end{aligned}
$$

The derivatives of $F_{2}(t)$ are

$$
F_{2}^{\prime}(t)=-24 \pi^{2} x^{3}+54 \pi^{3} x^{2}-44 \pi^{4} x+90 \pi^{2} x-90 x+14 \pi^{5}-45 \pi^{3}-45 \pi,
$$




$$
F_{2}^{\prime \prime}(x)=-72 \pi^{2} x^{2}+108 \pi^{3} x-44 \pi^{4}+90 \pi^{2}-90
$$

and

$$
F_{2}^{\prime \prime \prime}(x)=108 \pi^{3}-144 \pi^{2} x .
$$

From $F_{2}^{\prime \prime \prime}(x)<0$ for $\frac{3 \pi}{4}<x<\pi, F_{2}^{\prime \prime}(x)$ is strictly decreasing for $\frac{3 \pi}{4}<x<\pi$. By

$$
F_{2}^{\prime \prime}(\pi)=-90+90 \pi^{2}-8 \pi^{4} \cong 18.9917,
$$

we have $F_{2}^{\prime \prime}(x)>0$ for $\frac{3 \pi}{4}<x<\pi$ and $F_{2}^{\prime}(x)$ is strictly increasing for $\frac{3 \pi}{4}<x<\pi$. From

$$
F_{2}^{\prime}\left(\frac{3 \pi}{4}\right)=\frac{5}{4} \pi\left(-90+18 \pi^{2}+\pi^{4}\right) \cong 726.737,
$$

we have $F_{2}^{\prime}(x)>0$ for $\frac{3 \pi}{4}<x<\pi$ and $F_{2}(x)$ is strictly increasing for $\frac{3 \pi}{4}<x<\pi$. By

$$
F_{2}\left(\frac{3 \pi}{4}\right)=\frac{1}{128}\left(-19080 \pi^{2}+2760 \pi^{4}-23 \pi^{6}\right) \cong 456.446,
$$

we obtain $F_{2}(x)>0$ for $\frac{3 \pi}{4}<x<\pi$. Thus, we obtain $F(x)>0$ for $\frac{3 \pi}{4}<x<\pi$. The proof of Theorem 1.1 is complete.

Proof of Theorem 1.2. From $\pi \sqrt{\frac{2}{r-1}}<x<\pi$, we have

$$
r>\frac{x^{2}+2 \pi^{2}}{x^{2}}
$$

By the inequality (1.1), we have

$$
\begin{aligned}
\frac{1-\left(\frac{x}{\pi}\right)^{2}}{\sqrt{1+r\left(\frac{x}{\pi}\right)^{4}}}-\frac{\sin x}{x} & <\frac{1-\left(\frac{x}{\pi}\right)^{2}}{\sqrt{1+\left(\frac{x^{2}+2 \pi^{2}}{x^{2}}\right)\left(\frac{x}{\pi}\right)^{4}}}-\frac{\sin x}{x} \\
& =\frac{(\pi-x)(x+\pi)}{x^{2}+\pi^{2}}-\frac{\sin x}{x} \\
& <\frac{(\pi-x)(x+\pi)}{x^{2}+\pi^{2}}-\frac{\pi^{2}-x^{2}}{\pi^{2}+x^{2}} \\
& =0 .
\end{aligned}
$$

The proof of Theorem 1.2 is complete.

\section{Acknowledgements}

I would like to thank referee for him or her helpful suggestions and good advice. 


\section{References}

[1] A. Baricz, Redheffer type inequality for Bessel functions, J. Inequal. Pure Appl. Math., 8 (2007), no. 1 Art. 11, 6 pp.

[2] C. P. Chen, J. W. Zhao and F. Qi, Three inequalities involving hyperbolically trigonometric functions, RGMIA Res. Rep. Coll., 6 (2003), 437-443.

[3] L. Li and J. Zhang, A new proof on Redheffer-Williams' inequality, Far East J. Math. Sci., 56 (2011), $213-217$.

[4] J. L. Li and Y. L. Li, On the strengthened Jordan's inequality, J. Inequal. Appl., Art. ID 74328 (2007), 8 pp.

[5] J. L. Li, On a series of Erdós-Turán type, Analysis, 12 (1992), 315-317.

[6] K. Mehrez, Redheffer type inequalities for modified Bessel functions, Arab J. Math. Sci., 22 (2016), 38-42.

[7] R. Redheffer, P. Ungar, A. Lupas, et al., Problems and Solutions: Advanced Problems: 5642, 5665-5670, Amer. Math. Monthly, 76 (1969), 422-423.

[8] R. Redheffer and J. P. Williams, Solution of problem 5642, Amer. Math. Monthly, 76 (1969), $1153-1154$.

[9] L. Zhu and J. Sun, Six new Redheffer-type inequalities for circular and hyperbolic functions, Comput. Math. Appl., 56 (2008), 522-529.

[10] L. Zhu, Sharpening Redheffer-type inequalities for circular functions, Appl. Math. Lett., 22 (2009), $743-748$.

[11] L. Zhu, Extension of Redheffer type inequalities to modified Bessel functions, Appl. Math. Comput., 217 (2011), 8504-8506.

General Education, Ube National College of Technology, Tokiwadai 2-14-1, Ube, Yamaguchi 755-8555, Japan.

E-mail: yusuke@ube-k.ac.jp 